\title{
STABILITY PROPERTIES OF THE CLASS OF BANACH SPACES IN WHICH ALL MULTILINEAR FORMS ARE WEAKLY SEQUENTIALLY CONTINUOUS*
}

\author{
JESÚS M. F. CASTILLO, RICARDO GARCÍA \\ Departamento de Matemáticas, Universidad de Extremadura, Avenida de Elvas s/n, 06071 Badajoz, Spain \\ e-mail: castillo@unex.es,rgarcia@unex.es \\ and RAQUEL GONZALO \\ Facultad de Informática, Universidad Politécnica, Campus de Montegancedo, Boadilla del Monte, \\ 28660 Madrid, Spain \\ e-mail:rnonzalo@fi.upm.es
}

(Received 28 March, 2000; accepted 6 October 2000)

\begin{abstract}
A Banach space $X$ is said to be an $\mathcal{M}$-space if every continuous multilinear form on $X$ is weakly sequentially continuous. We study in this paper the stability properties of the class of $\mathcal{M}$-spaces.
\end{abstract}

2000 Mathematics Subject Classification. 46B20, 46B28, 46B08.

1. Introduction. A homogeneous scalar (continuous) polynomial on a Banach space $X$ is an application $p: X \rightarrow \mathbb{R}$ having the form $p(x)=M(x, \cdots, x)$ where $M$ is a multilinear (continuous) map on $X$. Conversely to what happens in the linear setting, multilinear forms or continuous polynomials are not usually continuous with respect to the weak topology. Perhaps the simplest example is the bilinear form that gives the inner product on a real Hilbert space (resp. the polynomial $\|\cdot\|^{2}$ ) which is obviously not weakly sequentially continuous since the canonical basis is weakly null.

Banach spaces in which all continuous multilinear forms (resp. polynomials) are weakly sequentially continuous have been called in [8] $\mathcal{M}$-spaces (resp. $\mathcal{P}$-spaces). It is not known if both classes coincide, although obviously $\mathcal{M}$-spaces are $\mathcal{P}$-spaces. Still more restrictive is the class of the hereditarily $\mathcal{M}$-spaces (resp. hereditarily $\mathcal{P}$-spaces): Banach spaces such that every subspace is an $\mathcal{M}$-space (resp. a $\mathcal{P}$-space). Gonzalo and Jaramillo have shown in [19] that hereditarily $\mathcal{P}$-spaces can be characterized as those Banach spaces that do not contain normalized sequences admitting lower $p$-estimates. (Recall that a sequence $\left(x_{n}\right)$ is said to satisfy a lower $p$ estimate [resp. an upper $p$-estimate] if for some constant $C>0$ and every finite sequence of scalars $\left(\lambda_{n}\right)$ one has $\left.\left\|\sum \lambda_{n} x_{n}\right\| \geq C\left(\sum\left|\lambda_{n}\right|^{p}\right)^{1 / p}\right)$ [resp. $\left.\leq\right]$.) Results in $[\mathbf{1 7}, \mathbf{1 8}]$ extend this characterization to multilinear forms showing that hereditarily $\mathcal{M}$ - and hereditarily $\mathcal{P}$-spaces coincide.

Recall that a space $X$ is said to have an upper p-estimate if every weakly null sequence contains a subsequence admitting an upper $p$-estimate. Reflexive spaces

*The research of the first and second authors has been supported in part by DGICYT project PB97-0377. That of the third author has been supported in part by DGICYT project PB96-0607. 
with upper $p^{*}$-estimates have been called $W_{p}$-spaces in [10]. A Banach space $X$ is said to have the Dunford-Pettis property if the bilinear form on $X \oplus X^{*}$ given by $(x, f) \rightarrow f(x)$ is weakly sequentially continuous.

The $\mathcal{M}$-spaces form a rather peculiar class: it contains the spaces with the Dunford-Pettis property [23] as well as spaces admitting, for every $p>1$, upper $p$ estimates (since this prevents the existence of sequences with lower $q$-estimates). Thus, spaces with the Dunford-Pettis property are $\mathcal{M}$-spaces, while spaces having upper $p$-estimates for all $p>1$ are hereditarily $\mathcal{M}$-spaces. It will be very useful to consider also $\mathcal{M}_{N}$-spaces (i.e., Banach spaces in which every $N$-linear form is weakly sequentially continuous).

The Dunford-Pettis property is stable under finite products, vector sums (see [5]), complemented subspaces and preduals. On the other hand, $W_{p}$-spaces are stable by finite products, vector sums (see [9]), subspaces and quotients. Nevertheless, an example in [8] shows that the product of two $\mathcal{M}$-spaces need not be an $\mathcal{M}$-space. Motivated by this, one should admit that the stability properties of the class of $\mathcal{M}$ spaces are, at least, intriguing.

The stability properties of the class of $\mathcal{M}$-spaces, including those obtained in this paper, can be summarized as follows:

1. Subspaces, quotients, duals or preduals of $\mathcal{M}$-spaces need not be $\mathcal{M}$ spaces. To be an $\mathcal{M}$-space is not stable by products, and thus this is not a threespace property. Nevertheless, if $E$ is an $\mathcal{M}$-space then $E \oplus E$ is an $\mathcal{M}$-space.

2. Complemented subspaces of $\mathcal{M}$-spaces are $\mathcal{M}$-spaces. Quotients of $\mathcal{M}$ spaces by subspaces not containing $l_{1}$ are $\mathcal{M}$-spaces.

3. If $\lambda$ is a Banach space with unconditional basis and $\lambda \oplus E$ is an $\mathcal{M}$-space not containing $l_{1}$ then $\lambda(X)$ is an $\mathcal{M}$-space. Alternatively, if either $\lambda$ or $X$ has the Dunford-Pettis property then $\lambda(X)$ is an $\mathcal{M}$-space. More precisely, the result is true for $\mathcal{M}_{N}$-spaces. There exist examples of $\mathcal{M}$-spaces $\lambda$ and $X$ such that $\lambda(X)$ is not an $\mathcal{M}$-space.

4. If $E^{* *}$ is an $\mathcal{M}$-space, then $E$ is an $\mathcal{M}$-space. The converse fails. However, if $E$ admits a certain local finite dimensional structure (in a sense to be made precise later), say $\left(E_{n}\right)$, and $l_{\infty}\left(E_{n}\right)$ is an $\mathcal{M}$-space then the ultrapowers of $E$ (and therefore all even duals of $E$ ) are $\mathcal{M}$-spaces.

5. The projective tensor product $E \otimes_{\pi} E$ of an $\mathcal{M}$-space need not be an $\mathcal{M}$ space. This answers a question in [8].

The class of $\mathcal{P}$-spaces has the same permanence properties, except that it is an open question whether $E \oplus E$ has to be a $\mathcal{P}$-space when $E$ is a $\mathcal{P}$-space.

2. Elementary stability properties. We pass to the proof of the results stated in 1 and 2. Since spaces with the Dunford-Pettis property (such as $l_{\infty}$ or $l_{1}$ ) are $\mathcal{M}$ spaces, while $l_{2}$ is not, it is clear that subspaces and quotients of $\mathcal{M}$-spaces are not necessarily $\mathcal{M}$-spaces. Nevertheless, it was established in [21, Prop. 2] that quotients of reflexive $\mathcal{P}$-spaces are $\mathcal{P}$-spaces. An analogous result for $\mathcal{P}$-spaces not containing $l_{1}$ (whose added difficulty is that one has to work with weakly Cauchy sequences instead of weakly convergent sequences) appears also mentioned as a note added in proof, while the proof is waived as "using Proposition 2.2 in [2]". Since the same is true for $\mathcal{M}$-spaces, let us state it as a lemma. 
Lemma 1. A multilinear form is weakly sequentially continuous if and only if it transforms weakly Cauchy sequences into convergent sequences.

This immediately gives the following result.

Proposition 1. Quotients of $\mathcal{M}$-spaces by subspaces not containing $l_{1}$ are $\mathcal{M}$-spaces.

Proof. Let $X$ be an $\mathcal{M}$-space and let $Q: X \rightarrow Z$ be a quotient map whose kernel does not contain $l_{1}$. By Lohman's lifting [22] weakly convergent sequences in $Z$ admit subsequences which are images by $Q$ of weakly Cauchy sequences in $X$ and, by Lemma $1, Z$ is an $\mathcal{M}$-space.

Tsirelson's original space $T^{*}$ is an $\mathcal{M}$-space since it admits, for all $p<\infty$, upper $p$-estimates. (See [11].) Moreover, its dual $T$ has a basis that admits, for all $q>1$, subsequences having lower $q$-estimates, which allows the construction of non-weakly sequentially continuous polynomials on $T$. This shows that neither the dual nor predual of an $\mathcal{M}$-space has to be an $\mathcal{M}$-space.

Multilinear forms cannot, as a rule, be extended from a subspace $Y$ of a Banach space $X$ to the whole space. When the multilinear forms on $Y$ can be extended to some $\mathcal{M}$-space then also $Y$ is an $\mathcal{M}$-space. This, and the Aron-Berner extension of a multilinear form to the bidual space, show that when the bidual $X^{* *}$ is an $\mathcal{M}$-space then the space $X$ is an $\mathcal{M}$-space; it also shows that complemented subspaces of $\mathcal{M}$ spaces are $\mathcal{M}$-spaces. The space $c_{0}\left(l_{2}(n)\right)$ has the Dunford-Pettis property and hence it is an $\mathcal{M}$-space, while its bidual $l_{\infty}\left(l_{2}(n)\right)$ contains a complemented copy of $l_{2}$ (see [5]) and thus it cannot be an $\mathcal{M}$-space.

3. The basic stability problem. In $[8]$ it was proved that neither the product nor the tensor product of two $\mathcal{M}$-spaces has to be an $\mathcal{M}$-space. Nevertheless, the following result holds.

Lemma 2. If $E$ is an $\mathcal{M}$-space then $E \oplus E$ is an $\mathcal{M}$-space.

Proof. An $n$-linear form on $E \oplus E$ can be thought of as $n^{n} n$-linear forms on $E$ : this is consequence of the algebraic (hence topological in the projective norm topology) isomorphism

$$
\bigotimes_{i=1}^{n}\left(E_{i} \oplus E_{i}\right)=\bigoplus_{i_{1}, \cdots, i_{n} \in\{1, \cdots, n\}}\left(E_{i_{1}} \otimes \cdots \otimes E_{i_{n}}\right)
$$

and the fact that the space of $m$-linear forms on $E$ is the dual space of the projective tensor product of $m$ copies of $E$. The proof is completely straightforward after that.

All this shows that the core of the difficulties for studying the stability of the class of $\mathcal{M}$-spaces lies in the existence of bilinear (multilinear) forms on $E \oplus F$ that cannot be reduced to bilinear forms on either $E$ or $F$. The following question appeared while working in [8] and seems to be of great importance for the understanding of the structure of the $\mathcal{M}$-spaces. 
Problem 1. Do $\mathcal{M}$-spaces and $\mathcal{P}$-spaces coincide?

Closely related, as we show next, is the following question.

Problem 2. Is $E \oplus E$ a $\mathcal{P}$-space when $E$ is a $\mathcal{P}$-space?

Lemma 3. If all m-homogenous polynomials on $E^{m}$ are weakly sequentially continuous, then all m-linear forms on E are weakly sequentially continuous.

Proof. Let $\sigma:\{1, \cdots, m\} \rightarrow\{1, \cdots, m\}$ be an $n$-cycle (for instance, $\sigma(j)=j+1$ and $\sigma(m)=1)$. If $M$ is an $m$-linear form on $E$ then

$$
\bar{M}\left[\left(x_{i}^{1}\right)_{i=1, \cdots, m}, \cdots,\left(x_{i}^{m}\right)_{i=1, \cdots, m}\right]=\sum_{k=0}^{k=m-1} M\left[x_{1}^{\sigma^{k}(1)}, x_{\sigma(1)}^{\sigma^{k+1}(1)}, \cdots, x_{\sigma^{m-1}(1)}^{\sigma^{k+m}(1)}\right]
$$

defines an $m$-linear form on $E^{m}$ such that

$$
\bar{M}\left[\left(x_{i}\right)_{i=1, \cdots, m}, \cdots,\left(x_{i}\right)_{i=1, \cdots, m}\right]=m M\left(x_{1}, \cdots, x_{m}\right) .
$$

The result easily follows after that.

A closely related decomposition for polynomials can be found in [1]. A consequence of Lemma 3 and Lemma 2 is that Problems 1 and 2 are equivalent.

Proposition 2. $\mathcal{M}$-spaces and $\mathcal{P}$-spaces coincide if and only if whenever $E$ is a $\mathcal{P}$-space then $E \oplus E$ is a $\mathcal{P}$-space.

Let us also remark that Dimant mentioned to us that Problem 2 can be shown to be equivalent to the following one that Dimant and Zalduendo pose in [15].

Problem 3. Does the space of $m$-homogeneous polynomials on $E$ have a basis if and only if the space of $m$-linear forms on $E$ has a basis?

4. Stability of vector sums. We pass to vector sums. Recall that given a Banach space $\lambda$ with an unconditional basis $\left(e_{n}\right)$ the vector sum space $\lambda(X)$ is defined as the space of all sequences $\left(x_{n}\right) \in X^{\mathbb{N}}$ such that $\left\|\left(x_{n}\right)\right\|_{\lambda(X)}=\left\|\left(\left\|x_{n}\right\|_{X}\right)\right\|_{\lambda}<+\infty$ endowed with the norm $\|\cdot\|_{\lambda(X)}$. It is a rather standard and usually interesting question to study the stability of a given property under vector sums. The reader may peruse [5,9], where it is proved that if both $\lambda$ and $E$ have the Dunford-Pettis property (resp. the hereditary Dunford-Pettis property) then so does $\lambda(X)$ or [13], devoted to the stability of the properties of containing $l_{p}$ or $c_{0}$.

Let us fix some notation. $\mathcal{L}(E, F)$ means the space of all operators acting between $E$ and $F$, while $\mathcal{K}(E, F)$ denotes the space of all compact operators. Given $A_{1}, \cdots, A_{N} N$-spaces, $\mathcal{L}^{N}\left(A_{1} \times \cdots \times A_{N}\right)$ denotes the space of $N$-linear forms on the product. $\mathcal{L}_{w s c}^{N}\left(A_{1} \times \cdots \times A_{N}\right)$ means the space of all weakly sequentially continuous $N$-linear forms. When $A_{1}=\cdots=A_{N}=X$ then we shall simply write $\mathcal{L}\left({ }^{N} X\right)$.

The next lemma extends one of the main results of [12] concerning natural tensor products with an $l_{p}$-space; we think it has an independent interest. 
Lemma 4. Let $X$ be a Banach space and let $\lambda$ be a Banach space with unconditional basis. Assume that $X$ and $\lambda$ do not contain $l_{1}$. Let $Z$ be a Banach space. If $\mathcal{L}(\lambda, Z)=\mathcal{K}(\lambda, Z)$ and $\mathcal{L}(X, Z)=\mathcal{K}(X, Z)$, then $\mathcal{L}(\lambda(X), Z)=\mathcal{K}(\lambda(X), Z)$

Proof. Let $T: \lambda(X) \rightarrow Z$ be an operator and let $\left(f_{n}\right)_{n}$ be a weakly null sequence in $\lambda(X)$. Since $\lambda(X)$ does not contain $l_{1}$ (see [20]) it is enough to show that $\left(T f_{n}\right)$ is norm null in $Z$.

It is possible to decompose $f_{n}=u_{n}+v_{n}$ with $u_{n}$ having finite support and $\lim \left\|v_{n}\right\|=0$. Hence, it is enough to show that $\lim \left\|T u_{n}\right\|=0$. Let $A_{1}$ be the support of $u_{1}$. We decompose $u_{n}=a_{n}^{1}+b_{n}^{1}$ with $a_{n}^{1}$ and $b_{n}^{1}$ having disjoint supports and $a_{n}^{1}$ having support contained in $A_{1}$. Since $\mathcal{L}(X, Z)=\mathcal{K}(X, Z)$ the same happens with $X^{k}$ and therefore $\lim \left\|T a_{n}^{1}\right\|=0$. Let $n(1)$ be such that $\left\|T a_{n(1)}^{1}\right\| \leq 2^{-1}$. We repeat the argument starting with $u_{n(1)}$ and relabel. In this way we obtain a sequence of elements $u_{n(k)}$ with supports $A_{k+1}$ such that $u_{n(k)}=a_{n(k)}^{k}+b_{n(k)}^{k}$ in which $a_{n(k)}^{k}$ and $b_{n(k)}^{k}$ have disjoint supports, $a_{n(k)}^{k}$ has support contained in $A_{k}$ and $\left\|T a_{n(k)}^{k}\right\| \leq 2^{-k}$. We show that $\lim \left\|T b_{n(k)}^{k}\right\|=0$, which concludes the proof. Observe that the vectors $b_{n(k)}^{k}$ are disjointly supported and thus the sequence $b_{n(k)}^{k}$ can be considered the image of a weakly null sequence $\left(\lambda_{k}\right)$ of $\lambda$ by some operator $L: \lambda \rightarrow \lambda(E)$ as we justify next in detail. After that, $T L: \lambda \rightarrow Z$ is compact and therefore $\lim \left\|T L \lambda_{k}\right\|=\lim \left\|T b_{n(k)}^{k}\right\|=$ 0 .

Let $f_{i}$ be norm one elements of $X^{*}$ chosen as follows. Let $B_{k}$ be the support of $b_{n(k)}^{k}$. If $i \in B_{k}$, then $f_{i}\left(b_{n(k)}^{k}(i)\right)=\left\|b_{n(k)}^{k}(i)\right\|_{X}$. The sequence $\left(f_{i}\right)_{i}$ induces an operator $F: \lambda(E) \rightarrow \lambda$ defined by $F\left(\left(x_{n}\right)_{n}\right)=\left(f_{n}\left(x_{n}\right)\right)_{n}$, which is continuous since $\left\|\left(f_{n}\left(x_{n}\right)\right)_{n}\right\|_{\lambda} \leq\left\|\left(\left\|x_{n}\right\|\right)_{n}\right\|_{\lambda}=\left\|\left(x_{n}\right)_{n}\right\|_{\lambda(X)}$. Therefore, the sequence

$$
\left.\left(F\left(b_{n(k)}^{k}\right)\right)_{k}=\left(\left(0, \ldots, 0,\left\|b_{n(k)}^{k}\left(\min B_{k}\right)\right\|_{X}, \cdots, \| b_{n(k)}^{k}\left(\max B_{k}\right)\right) \|_{X}, 0, \cdots\right)\right)_{k}
$$

is weakly null in $\lambda$. We set $\lambda_{k}=F\left(b_{n(k)}^{k}\right)$. The operator $L: \lambda \rightarrow \lambda(E)$ we are looking for comes is as follows: for $i \in B_{k}$,

$$
L\left(\left(\lambda_{n}\right)_{n}\right)(i)=\lambda_{i} \frac{\left.b_{n(k)}^{k}(i)\right)}{\left.\| b_{n(k)}^{k}(i)\right) \|_{X}}
$$

and 0 otherwise. The operator $L$ is continuous since

$$
\left\|L\left(\left(\lambda_{n}\right)_{n}\right)\right\|_{\lambda(X)} \leq\left\|\left(\left|\lambda_{n}\right|\right)_{n}\right\|_{\lambda} \leq K\left\|\left(\lambda_{n}\right)_{n}\right\|_{\lambda},
$$

where $K$ is the unconditionality constant of $\lambda$. Finally, it is clear that $L F\left(b_{n(k)}^{k}\right)=b_{n(k)}^{k}$.

Since it has been proved that every subsequence of $\left(u_{n}\right)_{n}$ contains a further subsequence whose image by $T$ converges to 0 , the same happens with the starting sequence $\left(u_{n}\right)_{n}$ and the proof is complete.

The following three lemmata clear the way for the proof of Theorem 1 .

Lemma 5. Let $V, W$ be Banach spaces. $\mathcal{L}\left(V, W^{*}\right)=\mathcal{K}\left(V, W^{*}\right)$ if and only if $\mathcal{L}\left(W, V^{*}\right)=\mathcal{K}\left(W, V^{*}\right)$. 
Proof. This is a consequence of the fact that an operator $T: V \rightarrow W^{*}$ is compact if and only if $T^{*}: W^{* *} \rightarrow V^{*}$ is compact; and, since $T^{*}$ is weak*-to weak ${ }^{*}$ continuous, $T^{*}$ is compact if and only if its restriction to the weak* dense part $V$ is compact.

The proof of the following lemma is contained in [14, Theorem 3.1].

Lemma 6. If $E$ is an $\mathcal{M}_{N}$-space and does not contain $l_{1}$ then $E \otimes_{\pi}{ }^{N \text { times }} \otimes_{\pi} E$ does not contain $l_{1}$.

Lemma 7. If $E$ and $F$ do not contain $l_{1}$, then all bilinear forms on $E \times F$ are weakly sequentially continuous if and only if all operators $E \rightarrow F^{*}$ are compact.

Proof. Recall first that bilinear forms on $E \times F$ can be identified with operators $E \rightarrow F^{*}$. Hence, $\mathcal{L}\left(X, X^{*}\right)=\mathcal{K}\left(X, X^{*}\right)$ is sufficient to make all bilinear forms on $X$ weakly sequentially continuous. As for the necessity, a careful reading of the proof of [8, Theorem 2.1] shows that if a bilinear form $B \in \mathcal{L}\left({ }^{2} X\right)$ is weakly sequentially continuous then its associated operator $\tau: X \rightarrow X^{*}$ transforms weakly null sequences $\left(x_{n}\right)$ of $X$ into bounded sequences $\left(\tau\left(x_{n}\right)\right)$ of $X^{*}$ such that for every weakly null sequence $\left(y_{k}\right)$ of $X$ one has

$$
\lim _{k \rightarrow \infty} \sup _{n \in N}\left|\tau\left(x_{n}\right)\left(y_{k}\right)\right|=0
$$

(recall that Emmanuele [16] calls a bounded set $A$ of $X^{*}$ an L-set if, for every weakly null sequence $\left(y_{k}\right)$ of $X$, one has

$$
\lim _{k \rightarrow \infty} \sup _{a \in A}\left|y_{k}(a)\right|=0 .
$$

Thus, we have shown that $\left(\tau\left(x_{n}\right)\right)_{n}$ is an L-set. Emmanuele shows in [16] that when $X$ does not contain $l_{1}$ the $L$-sets of $X^{*}$ are relatively compact; hence $\left(\tau\left(x_{n}\right)\right)_{n}$ is a relatively compact set, and the rest is routine.

A deeper analysis of the role of Emmanuele's $L$-sets in connection with weakly sequentially continuous multilinear forms can be found in [7].

Theorem 1. Let $X$ be a Banach space and let $\lambda$ be a Banach space with unconditional basis. Assume that $X$ and $\lambda$ do not contain $l_{1}$. If $\lambda \oplus X$ is an $\mathcal{M}_{N^{-}}$-space, then $\lambda(X)$ is an $\mathcal{M}_{N}$-space.

Proof. We should keep in mind for the rest of the proof that $\lambda(X)$ cannot contain $l_{1}$ as is proved in [20].

The case $N=2$ means to prove the implication

$$
\mathcal{L}\left(\lambda \oplus X, \lambda^{*} \oplus X^{*}\right)=\mathcal{K}\left(\lambda \oplus X, \lambda^{*} \oplus X^{*}\right) \Rightarrow \mathcal{L}\left(\lambda(X), \lambda(X)^{*}\right)=\mathcal{K}\left(\lambda(X), \lambda(X)^{*}\right) .
$$

After Lemma 4 it is enough to show that $\mathcal{L}\left(\lambda, \lambda(X)^{*}\right)=\mathcal{K}\left(\lambda, \lambda(X)^{*}\right)$ and $\mathcal{L}\left(X, \lambda(X)^{*}\right)=\mathcal{K}\left(X, \lambda(X)^{*}\right)$. We prove the first equality. Let $T: \lambda \rightarrow \lambda(X)^{*}$ be an operator. Since, by hypothesis, $\mathcal{L}\left(\lambda, \lambda^{*}\right)=\mathcal{K}\left(\lambda, \lambda^{*}\right)$ and $\mathcal{L}\left(X, \lambda^{*}\right)=\mathcal{K}\left(X, \lambda^{*}\right)$, Lemma 4 yields $\mathcal{L}\left(\lambda(X), \lambda^{*}\right)=\mathcal{K}\left(\lambda(X), \lambda^{*}\right)$. Therefore, $T^{*} \mid \lambda(X)$ is compact and, by Lemma 5 , so is $T^{*}$. The argument with $X$ is analogous. 
Assume that the case $N-1$ has been already proved. To prove that $\lambda(X)$ is an $\mathcal{M}_{N}$-space is clearly sufficient to obtain

$$
\mathcal{L}\left(\lambda(X), \mathcal{L}^{N-1}(\lambda(X))\right)=\mathcal{K}\left(\lambda(X), \mathcal{L}^{N-1}(\lambda(X))\right) .
$$

In view of Lemma 4 this is equivalent to obtaining simultaneously

and

$$
\mathcal{L}\left(\lambda, \mathcal{L}^{N-1}(\lambda(X))\right)=\mathcal{K}\left(\lambda, \mathcal{L}^{N-1}(\lambda(X))\right)
$$

$$
\mathcal{L}\left(X, \mathcal{L}^{N-1}(\lambda(X))\right)=\mathcal{K}\left(X, \mathcal{L}^{N-1}(\lambda(X))\right)
$$

Consider for instance the second equality. By the induction assumption, $\lambda(X)$ is an $\mathcal{M}_{N-1}$-space and thus, by Lemma $6, \lambda(X) \otimes_{\pi_{*}{ }^{N-1} \text {. times }} \otimes_{\pi} \lambda(X)$ does not contain $l_{1}$. Since $\mathcal{L}^{N-1}(\lambda(X))=\left(\lambda(X) \otimes_{\pi}{ }^{N-1} .{ }^{\text {times }} \otimes_{\pi} \lambda(X)\right)^{*}$, Lemma 7 asserts that the desired equality is equivalent to showing that

$$
\mathcal{L}^{N}\left(X \times \lambda(X)^{N-1}\right)=\mathcal{L}_{w s c}^{N}\left(X \times \lambda(X)^{N-1}\right) .
$$

This is in turn equivalent to

$$
\mathcal{L}\left(\lambda(X), \mathcal{L}^{N-1}\left(X \times \lambda(X)^{N-2}\right)\right)=\mathcal{K}\left(\lambda(X), \mathcal{L}^{N-1}\left(X \times \lambda(X)^{N-2}\right)\right) .
$$

Once again Lemma 4 makes this equivalent to obtaining simultaneously

and

$$
\mathcal{L}\left(\lambda, \mathcal{L}^{N-1}\left(X \times \lambda(X)^{N-2}\right)\right)=\mathcal{K}\left(\lambda, \mathcal{L}^{N-1}\left(X \times \lambda(X)^{N-2}\right)\right)
$$

$$
\mathcal{L}\left(X, \mathcal{L}^{N-1}\left(X \times \lambda(X)^{N-2}\right)\right)=\mathcal{K}\left(X, \mathcal{L}^{N-1}\left(X \times \lambda(X)^{N-2}\right)\right)
$$

After repeating $N$-times this process of "shifting $\lambda$ and $X$ to the left" one obtains that the starting equality becomes equivalent to the $2^{N}$ equalities

$$
\mathcal{L}^{N}\left(A_{1} \times{ }^{N-1} \cdot{ }^{\text {times }} \times A_{N}\right)=\mathcal{L}_{w s c}^{N}\left(A_{1} \times{ }^{N-1} \cdot{ }^{\text {times }} \times A_{N}\right)
$$

in which $A_{i}$ means either $X$ or $\lambda$. Also this is implied by the hypothesis " $\lambda \oplus X$ is an $\mathcal{M}_{N}$-space".

COROllary 1. Let $X$ be a Banach space and let $\lambda$ be a Banach space with unconditional basis. Assume that $X$ and $\lambda$ do not contain $l_{1}$. If $\lambda \oplus X$ is an $\mathcal{M}$-space then $\lambda(X)$ is an $\mathcal{M}$-space.

A question suggested by the previous proof (observe that the hypothesis of not containing $l_{1}$ is necessary) is as follows.

Problem 4. Assume that $E \otimes_{\pi} E$ does not contain $l_{1}$. Does $\mathcal{L}(E, Z)=\mathcal{K}(E, Z)$ imply $\mathcal{L}\left(E \otimes_{\pi} E, Z\right)=\mathcal{K}\left(E \otimes_{\pi} E, Z\right)$ ?

Let us show that the additional hypothesis about $\lambda \oplus X$ in Proposition 1 cannot be omitted. As we already mentioned, one of the main examples in [8] is a Lorentz sequence space $d(w, 1)$ such that both $d(w, 1)$ and its natural predual $d_{*}(w)$ are 
$\mathcal{M}$-spaces while the canonical bilinear form on $d_{*}(w) \oplus d(w, 1)$ is not weakly sequentially continuous (i.e., $d_{*}(w)$ has not the Dunford-Pettis property). Since those two spaces have symmetric basis, one can construct the vector sums $d(w, 1)\left(d_{*}(w)\right)$ and $d_{*}(w)(d(w, 1))$, which cannot be $\mathcal{M}$-spaces.

We investigate now the role of the Dunford-Pettis property. The following result shows that the example in $[8]$ is "sharp".

Proposition 3. If $X$ is an $\mathcal{M}$-space and $Y$ a space with the Dunford-Pettis property, then $X \oplus Y$ is an $\mathcal{M}$-space.

Proof. A bilinear form $B$ on $X \oplus Y$ can be reduced to four elements:

- a bilinear form $B_{X}$ on $X$ given by

$$
B_{X}(x, a)=B((x, 0),(a, 0)),
$$

- a bilinear form $B_{Y}$ on $Y$, given by

$$
B_{Y}(y, b)=B((0, y),(0, b)),
$$

- a linear operator $L_{X}: X \rightarrow Y^{*}$ given by

$$
L_{X}(x)(b)=B((x, 0),(0, b))
$$

and

- a linear operator $L_{Y}: Y \rightarrow X^{*}$ given by

$$
L_{Y}(y, a)=B((0, y),(a, 0))
$$

in the form

$$
B((x, y),(a, b))=B_{X}(x, a)+L_{X}(x)(b)+L_{Y}(y)(a)+B_{Y}(y, b) .
$$

Quite clearly, if $\left(\left(x_{n}, y_{n}\right),\left(a_{n}, b_{n}\right)\right)_{n}$ is a weakly null sequence in $(X \oplus Y) \oplus$ $(X \oplus Y)$ then $B\left(\left(x_{n}, y_{n}\right),\left(a_{n}, b_{n}\right)\right)$ converges to zero: the sequence $\left(L_{X}\left(x_{n}\right)\left(b_{n}\right)\right)_{n}$ tends to zero since $Y$ has the Dunford-Pettis property; analogously, identifying the points in $X$ with their canonical images in $X^{* *}$, one obtains that the sequence $\left(L_{Y}\left(y_{n}\right)\left(a_{n}\right)\right)_{n}$ converges to zero; finally, the sequences $\left(B_{X}\left(x_{n}, a_{n}\right)\right)_{n}$ and $\left(B_{Y}\left(y_{n}, b_{n}\right)\right)_{n}$ converge to zero since $X$ and $Y$ are $\mathcal{M}$-spaces.

The proof for an $m$-linear form is analogous: let $D$ be a set of indices $\alpha$ such that there exists a decomposition $\left(x_{\alpha}, y_{\alpha}\right)$ of $m$ in integers, including 0 , such that $x_{\alpha}+y_{\alpha}=m$. An $m$-linear form $M$ on $X \oplus Y$ can be decomposed as $M=\sum_{\alpha \in D} M_{\alpha}$ where $M_{\alpha}$ is a $x_{\alpha}$-linear form on $X$ taking values on the space of $y_{\alpha}$-linear forms on $Y$. Since finite products of spaces with the Dunford-Pettis property have the Dunford-Pettis property and $X$ is an $\mathcal{M}$-space, reasoning as in Ryan's proof [23] and, following the pattern of the bilinear case, one can show that all the components $M_{\alpha}$ are weakly sequentially continuous.

When either $\lambda$ or $X$ has the Dunford-Pettis property then not only the hypothesis on $\lambda \oplus X$ can be dropped, one can also discard the hypothesis of not containing $l_{1}$. 
TheOREM 2. Let $\lambda$ be a Banach sequence space with an unconditional basis and let $X$ be a Banach space. Assume that both $\lambda$ and $X$ are $\mathcal{M}$-spaces. If either $\lambda$ or $X$ have the Dunford-Pettis property then $\lambda(X)$ is an $\mathcal{M}$-space.

Proof. Assume that it is $\lambda$ which has the Dunford-Pettis property. We prove first that bilinear forms on $\lambda(X)$ are weakly sequentially continuous and then proceed by induction.

Let $B$ be a bilinear form on $\lambda(X)$ and let $\left(f_{n}\right)_{n}$ and $\left(g_{n}\right)_{n}$ be weakly null sequences in $\lambda(X)$. We follow the same notation as in Lemma 4 . We start by writing $f_{n}=u_{n}+v_{n}$ and $g_{n}=w_{n}+y_{n}$, where $\lim \left\|v_{n}\right\|=\lim \left\|y_{n}\right\|=0$ while $u_{n}$ and $w_{n}$ have finite support. Since

$$
B\left(f_{n}, g_{n}\right)=B\left(u_{n}, w_{n}\right)+B\left(u_{n}, y_{n}\right)+B\left(v_{n}, w_{n}\right)+B\left(v_{n}, y_{n}\right),
$$

it only has to be shown that $\lim \left\|B\left(u_{n}, w_{n}\right)\right\|=0$. We decompose $u_{n}=a_{n}^{1}+b_{n}^{1}$ and $w_{n}=c_{n}^{1}+d_{n}^{1}$ as in Lemma 4 . This gives

$$
B\left(u_{n}, w_{n}\right)=B\left(a_{n}^{1}, c_{n}^{1}\right)+B\left(a_{n}^{1}, d_{n}^{1}\right)+B\left(b_{n}^{1}, c_{n}^{1}\right)+B\left(b_{n}^{1}, d_{n}^{1}\right) .
$$

Since $X$ is an $\mathcal{M}$-space, so are the finite products $X^{k}$. Since $\left(B\left(\cdot, c_{n}^{1}\right)\right)_{n}$ is a weakly null sequence in $\lambda(X)^{*}$, its restriction to the finite product $X^{k}$ in which the supports of $u_{1}$ and $w_{1}$ are contained must be weakly sequentially continuous. Thus,

$$
\lim \left\|B\left(a_{n}^{1}, c_{n}^{1}\right)\right\|=0 .
$$

It is therefore possible to obtain some index $n(1)$ such that, for $j \geq n(1)$ one has $\left\|B\left(a_{j}^{1}, c_{j}^{1}\right)\right\| \leq 2^{-1}$. Repeating the reasoning with $u_{n(1)}$ and $w_{n(1)}$, and then diagonalizing, we obtain a subsequence $(n(k))_{k}$ such that $\left\|B\left(a_{n(k)}^{k}, c_{n(k)}^{k}\right)\right\| \leq 2^{-k}$; and thus

$$
\lim \left\|B\left(a_{n(k)}^{k}, c_{n(k)}^{k}\right)\right\|=0 .
$$

Let us relabel the sequences so that

$$
B\left(u_{n}, w_{n}\right)=B\left(a_{n}, c_{n}\right)+B\left(a_{n}, d_{n}\right)+B\left(b_{n}, c_{n}\right)+B\left(b_{n}, d_{n}\right),
$$

where $\left(b_{n}\right)_{n}$ and $\left(d_{n}\right)_{n}$ are images of weakly null sequences, say $\left(\lambda_{n}\right)_{n}$ and $\left(\mu_{n}\right)_{n}$, of $\lambda$ by some operators, say $\tau_{b}$ and $\tau_{d}$. Moreover, the preceding argument shows that there is no loss of generality in assuming that

$$
\lim B\left(a_{n}, c_{n}\right)=0 .
$$

Since $\left(a_{n}\right)_{n}$ and $\left(c_{n}\right)_{n}$ are weakly null sequences in $\lambda(X)$, then $\left(B\left(a_{n}, \tau_{d}(\cdot)\right)\right)_{n}$ and $\left(B\left(\tau_{b}(\cdot), c_{n}\right)_{n}\right.$ are weakly null sequences in $\lambda^{*}$ while $\left(B\left(\tau_{b}(\cdot), \tau_{d}(\cdot)\right)\right)_{n}$ is a bilinear form on $\lambda$. The Dunford-Pettis property of $\lambda$ yields

$$
\begin{aligned}
& \lim B\left(a_{n}, d_{n}\right)=\lim B\left(a_{n}, \tau_{d}\left(\mu_{n}\right)\right)=0, \\
& \lim B\left(b_{n}, c_{n}\right)=\lim B\left(\tau_{b}\left(\lambda_{n}\right), c_{n}\right)=0, \\
& \lim B\left(b_{n}, d_{n}\right)=\lim B\left(\tau_{b}\left(\lambda_{n}\right), \tau_{d}\left(\mu_{n}\right)\right)=0 .
\end{aligned}
$$


This concludes the proof that $B$ is weakly sequentially continuous and $\lambda(X)$ is an $\mathcal{M}_{2}$-space when $\lambda$ has the Dunford-Pettis property.

The proof when $X$ has the Dunford-Pettis property is analogous. In this case one has to consider that $B\left(\cdot, d_{n}\right)$ and $B\left(\cdot, c_{n}\right)$ form weakly null sequences in $\lambda(X)^{*}$; thus, when restricted to the finite products $\left(X^{*}\right)^{k}$ they must transform weakly null sequences into null sequences. This is what makes $\lim B\left(a_{n}, c_{n}\right)=\lim B\left(b_{n}, d_{n}\right)=0$. The equality $B\left(b_{n}, d_{n}\right)=B\left(\tau_{b}\left(\lambda_{n}\right), \tau_{d}\left(\mu_{n}\right)\right)$ shows that the last sequence is described by the action of a bilinear form on $\lambda$, and must therefore converge to zero.

The proof for trilinear forms and, in general, the inductive step, can be easily obtained recalling that, given an $m$-linear form $M$ on a Banach space $E$ and points $x, z \in E^{m}$, one has the decomposition

$$
M(x+z)=M(x)+M_{1}(x ; z)+M(z),
$$

where, for each $x \in E, M_{1}(x ; z)$ is a sum of $n$-linear forms on $E$ with $1 \leq n<m$.

It only remains to pose the following question.

Problem 5. Assume that $\lambda \oplus X$ is an $\mathcal{M}$-space. Is $\lambda(X)$ an $\mathcal{M}$-space?

The guess of the first author is that the answer is no, while the second author conjectures a positive answer.

5. $l_{\infty}$-sums and local structure. A rather more difficult case is that of $l_{\infty}$-sums of $\mathcal{M}$-spaces. In general, no positive result can be expected since it was already mentioned that the $l_{\infty}$-sum of finite dimensional Hilbert spaces contains a complemented copy of $l_{2}$ and thus it cannot be an $\mathcal{M}$-space. Curiously enough, this is the same example that shows that the bidual of an $\mathcal{M}$-space need not be an $\mathcal{M}$-space. We give now a partial result connecting both results.

Given a sequence $\left(E_{n}\right)$ of finite-dimensional normed spaces we say that a Banach space $E$ admits the family $\left(E_{n}\right)$ as local structure if for some $c>0$ every finite dimensional subspace $A \subset E$ is contained in some finite dimensional subspace $B \subset E$ that is $c$-isomorphic to some $E_{n}$.

Proposition 4. Assume that E has some local structure $\left(E_{n}\right)$. If $l_{\infty}\left(E_{n}\right)$ is an $\mathcal{M}$ space then all ultrapowers of $E$ (and hence all even duals) are $\mathcal{M}$-spaces.

Proof. Ultrapowers of $E$ have the same local structure as $E$, and even duals of $E$ are 1-complemented in some ultrapower. Now, let $E_{\mathcal{U}}$ be an ultrapower of $E$ and let $Y$ be a separable subspace of $E_{\mathcal{U}}$. Necessarily, $Y$ is contained in a subspace of $E_{\mathcal{U}}$ having the form $\overline{\cup_{j} E_{n}(j)}$ (up to an isomorphism). Let us prove that $\overline{\cup_{n} E_{n}}$ is an $\mathcal{M}$-space; this suffices.

Let $\left(x_{n}^{i}\right)_{n \in N}, 1 \leq i \leq r$, be a finite number of normalized weakly null sequences in $\overline{\cup_{n} E_{n}}$. There is no loss of generality in assuming that $x_{n}^{i} \in E_{n}$, for all $1 \leq i \leq r$. Let $m: \overline{\cup_{n} E_{n}} \rightarrow \mathbb{R}$ be an $r$-linear form. We define an $r$-linear form $M: l_{\infty}\left(\bar{E}_{n}\right) \rightarrow \mathbb{R}$ by means of

$$
M\left(\left(u_{n}^{1}\right)_{n}, \cdots,\left(u_{n}^{r}\right)_{n}\right)=\lim _{\mathcal{U}(n)} m\left(u_{n}^{1}, \cdots, u_{n}^{r}\right)
$$


For fixed $1 \leq i \leq r$, the points $v_{k}^{i} \in l_{\infty}\left(E_{n}\right)$ defined by $v_{k}^{i}(j)=0$ if $j<k$ and $v_{k}^{i}(j)=x_{k}^{i}$ if $j \geq k$ have norm 1 and form a weakly null sequence, as is easily verified. Since $l_{\infty}\left(E_{n}\right)$ is an $\mathcal{M}$-space and $M\left(v_{k}^{1}, \cdots, v_{k}^{r}\right)=m\left(x_{k}^{1}, \cdots, x_{k}^{r}\right)$, it follows that $\overline{\cup_{n} E_{n}}$ is an $\mathcal{M}$-space.

REMARK. We have proved slightly more than announced: every separable subspace of $E_{\mathcal{U}}$ is contained in a separable $\mathcal{M}$-subspace of $E_{\mathcal{U}}$.

6. Lack of stability by projective tensor products. The question of whether the projective tensor product of two $\mathcal{M}$-spaces has to be an $\mathcal{M}$-space is implicit in [8], where it is shown that the projective tensor product of two polynomially null sequences need not be even weakly null. It is not hard to deduce from the results presented there that the projective tensor product of two $\mathcal{M}$-spaces need not be an $\mathcal{M}$-space. However, there remains the question of whether the tensor product $E \otimes_{\pi} E$ is an $\mathcal{M}$-space when $E$ is an $\mathcal{M}$-space. A negative answer was shown to the first author during the July 1999 Conference on Banach spaces at Murcia by I. Villanueva. The following result can be deduced from the results in [3]. (See, in particular Theorem 2.2 of [3].)

Proposition 5. Let $K$ and $S$ be two compact Hausdorff spaces. Then $C(K) \otimes_{\pi} C(S)$ is an $\mathcal{M}$-space if and only if both $K$ and $S$ are scattered.

The key of the argument is to show (Lemma 2.1 in [3]) that the projective tensor product of a weakly null and a bounded sequence in $C(K)$-spaces is still weakly null. Now, if $K$ is not scattered then there exists a quotient map $q: C(K) \rightarrow l_{2}$; taking a bounded sequence $\left(f_{n}\right)$ in $C(K)$ such that $q\left(f_{n}\right)=e_{n}$, the canonical basis of $l_{2}$ and taking a weakly null sequence $\left(g_{n}\right)$ in $C(S)$ and a bounded sequence $\left(g_{n}^{*}\right)$ in $C(S)^{*}$ such that $g_{n}^{*}\left(g_{n}\right)=1$ one gets that the 4-linear form $C: C(K) \times C(S) \times C(K) \times$ $C(S) \rightarrow \mathbb{R}$ defined by $C(f, g, h, j)=\sum_{n} q(f)(n) g_{n}^{*}(g) q(h)(n) g_{n}^{*}(j)$ induces a bilinear form $B: C(K) \otimes_{p} i C(S) \times C(K) \otimes_{\pi} C(S) \rightarrow \mathbb{R}$ that is not weakly sequentially continuous since $B\left(\left(f_{n} \otimes g_{n}\right),\left(f_{n} \otimes g_{n}\right)\right)=C\left(f_{n}, g_{n}, f_{n}, g_{n}\right)=1$.

\section{REFERENCES}

1. J. M. Ansemil and K. Floret, The symmetric tensor product of a direct sum of locally convex spaces, Studia Math. 129 (1998), 285-295.

2. R. Aron, C. Herves and M. Valdivia, Weakly continuous mappings on Banach spaces, J. Funct. Anal. 52 (1983), 189-204.

3. F. Bombal and I. Villanueva, On the Dunford-Pettis property of the tensor product of $C(K)$-spaces, Proc. Amer. Math. Soc. 129 (2000), 1359-1363. 272.

4. J. Bourgain, On the Dunford-Pettis property, Proc. Amer. Math. Soc. 81 (1981), 265-

5. J. M. F. Castillo and M. González, New results on the Dunford-Pettis property, Bull. London Math. Soc. 27 (1995), 599-605.

6. J. M. F. Castillo and M. González, Three-space problems in Banach space theory, Lecture Notes in Mathematics No. 1667 (Springer-Verlag, 1997).

7. J. M. F. Castillo and R. García, $L$-sets and the Pelczynski-Pitt theorem, preprint, 1999.

8. J. M. F. Castillo, R. García and R. Gonzalo, Banach spaces in which all multilinear forms are weakly sequentially continuous, Studia Math. 136 (1999), 121-145. 
9. J. M. F. Castillo and F. Sánchez, Upper $l_{p}$-estimates in vector sequence spaces, with some applications, Math. Proc. Cambridge Philos. Soc. 113 (1993), 329-334.

10. J. M. F. Castillo and F. Sánchez, Weakly p-compact, p-Banach-Saks and superreflexive Banach spaces, J. Math. Anal. Appl. 185 (1994), 256-261.

11. J. M. F. Castillo and F. Sánchez, Remarks on some basic properties of Tsirelson's space, Note di Mat. 13 (1993), 117-122.

12. J. M. F. Castillo and J. A. López Molina, Operators defined on projective and natural tensor products, Michigan Math. J. 40 (1993), 411-415.

13. P. Cembranos and J. Mendoza, Banach spaces of vector-valued functions, Lecture Notes in Math. No. 1676 (Springer-Verlag, 1997).

14. Y. S. Choi and S. G. Kim, Polynomial properties of Banach spaces, J. Math. Anal. Appl. 190 (1995), 203-210.

15. V. Dimant and I. Zalduendo, Bases in spaces of multilinear forms over Banach spaces, J. Math. Anal. Appl. 200 (1996), 548-566.

16. G. Emmanuele, A dual characterization of spaces not containing $l_{1}$, Bull. Polish Acad. Sci. Math. 34 (1986), 155-160.

17. R. Gonzalo, Multilinear forms, subsymmetric polynomials and spreading models on Banach spaces, J. Math. Anal. Appl. 202 (1996), 379-397.

18. R. Gonzalo and J. A. Jaramillo, Compact polynomials between Banach spaces, Proc. Roy. Irish Acad. Sect. A 95 (1995), 321-341.

19. R. Gonzalo and J. A. Jaramillo, Smoothness and estimates of sequences in Banach spaces, Israel J. Math. 89 (1993), 321-341.

20. F. Hernández and V. Peirats, A remark on sequence F-spaces $\lambda(E)$ containing a copy of $l_{p}$, Bull. Polish Acad. Sci. Math. 34 (1986), 295-299.

21. J. A. Jaramillo and A. Prieto, Weak polynomial convergence on a Banach space, Proc. Amer. Math. Soc. 118 (1993), 463-468.

22. R. H. Lohman, A note on Banach spaces containing $l_{1}$, Canad. Math. Bull. 19 (1976), $365-367$.

23. R. Ryan, Dunford-Pettis properties, Bull. Polish Acad. Sci. Math. 27 (1979), 373-379. 